\title{
Macrofauna Acuática de la Quebrada Santa Inés, Subcuenca del Río Yeguare, Honduras
}

\author{
López, Lucía Isabel ${ }^{1}$, Pablo Gutiérrez ${ }^{2}$ y José, M. Mora ${ }^{3}$
}

\begin{abstract}
Resumen. La quebrada Santa Inés forma parte de la subcuenca del río Yeguare ubicada en los departamentos de Francisco Morazán y El Paraíso, Honduras. La quebrada abastece de agua a varias comunidades. Se estudió la composición de la comunidad de macroinvertebrados acuáticos de la quebrada Santa Inés, para lo cual se muestrearon tres estaciones. Se recolectaron 3,525 individuos en la quebrada pertenecientes a 55 familias. El grupo dominante en la quebrada fue Ephemeroptera, orden generalmente abundante en ríos de montaña. En cuanto a la estructura trófica, los depredadores fueron constantes a lo largo de la corriente, mientras que los filtradores fue el segundo grupo dominante en la quebrada con $25 \%$ de los individuos. La quebrada Santa Inés contiene una alta complejidad estructural, donde se encuentran representados todos los grupos tróficos de macroinvertebrados acuáticos posibles en un sistema ribereño. La geografía de la microcuenca de la quebrada Santa Inés, el uso actual del suelo y la comunidad de macroinvertebrados acuáticos que se encuentra en la quebrada la hacen un sujeto ideal de estudio bajo el concepto del continuo ribereño.
\end{abstract}

Palabras clave: Biodiversidad, continuo ribereño, macroinvertebrados acuáticos, microcuenca.

\section{Aquatic Macroinvertebrates of Quebrada Santa Inés, Micro Basin of the Yeguare River, Honduras}

\begin{abstract}
Santa Ines is a stream of the Yeguare River sub basin, located in the departments of Francisco Morazán and El Paraíso, Honduras. Santa Ines supplies water to several human communities. Three stations were sampled to study the macro invertebrate community composition of the Santa Ines stream. A group of 3,525 individuals was collected in the stream belonging to 55 families. The dominant group was Ephemeroptera, an order generally abundant in mountain streams. On the trophic structure, predators were constant along the stream, while filter-feeding insects were the second dominant group in Santa Ines with $25 \%$ of the individuals. Santa Ines contains a high structural complexity, where all possible trophic groups of aquatic macroinvertebrates in a river system are represented. The micro basin geography of the Santa Ines stream, the current land use and the community of aquatic macroinvertebrates found in the stream makes it an ideal study subject under the River Continuum Concept.
\end{abstract}

Key words: Biodiversity, aquatic macroinvertebrates, micro basin, River Continuum Concept.

\section{Introducción}

La cantidad y la calidad del agua son temas de interés debido a la demanda cada vez mayor de este recurso. Los parámetros fisicoquímicos son los métodos de más amplia utilización para evaluar la calidad del agua. Sin embargo, los organismos que habitan en los cursos de agua pueden ser utilizados para monitorear las alteraciones en estos ecosistemas (Alba-Tercedor 1996). Lo anterior es posible ya que las comunidades de organismos responden a las alteraciones debido a que estos presentan diferente sensibilidad a las sustancias contaminantes (WalterosRodríguez y Paiba-Alzate 2010). Debido a ello, los organismos acuáticos, como los macroinvertebrados, pueden ser utilizados como indicadores biológicos.

La alta biodiversidad de organismos en los cursos de agua está representada por peces, anfibios, mamíferos, algas y aves (Ramachandra y Solanki 2007). Empero, el grupo más importante, por su papel en el ambiente y que a la vez tiene una distribución más amplia en estos sistemas, es el de los macroinvertebrados acuáticos. Desde el siglo pasado, los macroinvertebrados han sido ampliamente

\footnotetext{
${ }^{1}$ Consultora Ambiental, Zamorano, Honduras, correo electrónico: luciaisa2@gmail.com

${ }^{2}$ Escuela de Biología, Universidad de Costa Rica, correo electrónico: gutifp@ gmail.com

${ }^{3}$ Centro Zamorano de Biodiversidad, Carrera de Ingeniería en Ambiente y Desarrollo, Escuela Agrícola Panamericana, Zamorano, Honduras, correo electrónico: jmora@zamorano.edu
} 
estudiados en Europa y el norte de América. Sin embargo, en los trópicos la taxonomía es casi desconocida para la mayoría de especies y apenas se comienza por entender sus funciones en los ecosistemas (Roldán 2003, Molina et al. 2008).

El grupo más abundante de los macroinvertebrados acuáticos son los insectos. Éstos cumplen diversos papeles y funciones en el medio acuático tales como el rompimiento de la materia orgánica, el reciclaje de nutrientes y son eslabones en las redes tróficas (Smock 1996, Roldán 1992), entre otras funciones. Existen insectos (así como otros grupos de macroinvertebrados) que tienen la capacidad de tolerar la contaminación por lo que pueden ser usados en la bioindicación (Helawell 1986, Rosenberg et al. 1986, Resh et al. 1996, Roldán 1999).

Los insectos acuáticos en Honduras han sido poco estudiados, pero destacan los trabajos de Vidal (1930), Packer (1966) y Bass (1993). Otros trabajos taxonómicos incluyen descripciones de especies hondureñas: Harris y Holzenthal (1999), Wiersema y McCafferty (2000), Prather (2003), Estévez y Polhemus (2006) y Blanhnik y Holzenthal (2008). Son pocos los trabajos realizados con macroinvertebrados acuáticos en sitios específicos o sistemas particulares (Cruz et al. 1987, García y Jimenez 2006, Arcos et al. 2005, Fenoglio et al. 2008, López 2008).

El propósito del presente trabajo fue determinar la composición de la comunidad de macroinvertebrados acuáticos de la quebrada Santa Inés, en la subcuenca del río Yeguare. Esta información es útil desde el punto de vista del conocimiento de la biodiversidad de la zona. También es útil para ayudar en la determinación de la calidad del agua de dicha quebrada. Esta agua es utilizada por varias comunidades, incluida la Escuela Agrícola Panamericana, El Zamorano.

\section{Materiales y Métodos}

La Quebrada Santa Inés se encuentra en los departamentos de Francisco Morazán y El Paraíso en la región centro sur de Honduras (Figura 1). Abarca 1,976 ha y abastece de agua a las comunidades de Lavanderos, Santa Inés, Santa Rosa, El Guayabo, El Hondable, Matahambre y Zamorano (Herrera 2001).
La precipitación promedio anual en Santa Inés es de unos $1,000 \mathrm{~mm}$ en las partes bajas y cerca de 4,000 $\mathrm{mm}$ en las partes altas. La temperatura promedio anual es de $6^{\circ} \mathrm{C}$ en las zonas altas y $24^{\circ} \mathrm{C}$ en las bajas (Renderos 2001). En la microcuenca Santa Inés el bosque natural abarca 935 ha y el resto (más de 1,000 ha) son áreas de cultivos agrícolas, entre los que sobresalen el frijol y maíz. Las hortalizas, tales como tomate, cebolla y papa, también son comunes en el área. Aunque en el área de la microcuenca se distinguen tres zonas de vida del sistema Holdridge (1971) los sitios muestreados se ubican dentro de la zona de vida del bosque húmedo subtropical (bh-S).

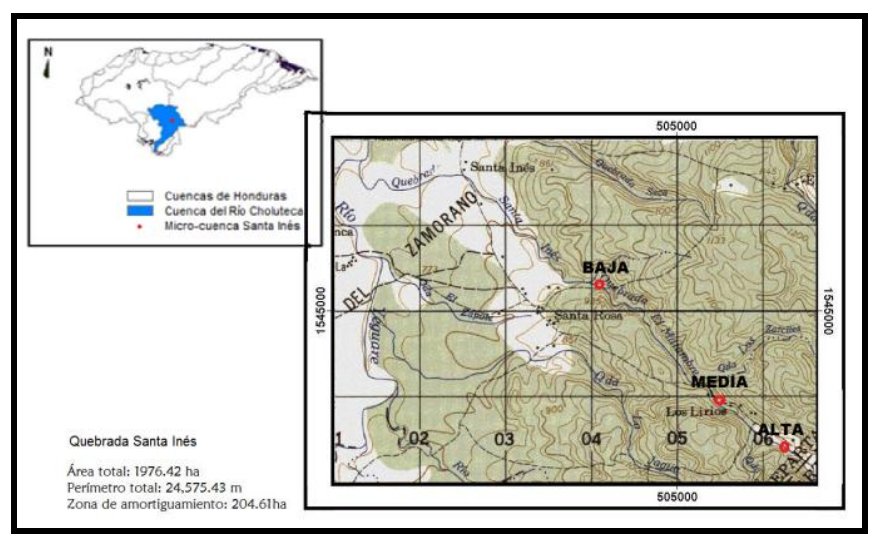

Figura 1. Estaciones de muestreo (alta, media y baja) de organismos acuáticos en la Quebrada Santa Inés, Cuenca del río Choluteca, Honduras.

Se muestreó siete veces en tres estaciones definidas en el curso principal de la Quebrada Santa Inés, de febrero a agosto de 2007. Las estaciones están ubicadas en la parte alta, media y baja de la quebrada Santa Inés (Cuadro 1). Cada estación se ubicó geográficamente con un posicionador global portátil, GPS $\left(\right.$ GARMIN $\left.^{\mathrm{TM}}\right)$.

En cada estación se recolectaron los macroinvertebrados acuáticos con una red manual de $20 \mathrm{~cm}$ de diámetro y $0.5 \mathrm{~mm}$ de poro. En cada estación se removió el lecho de la quebrada (hojarasca y rocas) y con la red de mano y pinzas entomológicas se recolectaron todos los macroinvertebrados atrapados en la red. Este procedimiento se realizó por períodos 
de 15 minutos hasta completar una hora en cada sitio. Los macroinvertebrados se depositaron en frascos plásticos con alcohol al $70 \%$ para preservarlos. Cada frasco fue etiquetado con el número de la estación y fecha de muestreo. La separación, identificación y conteo se efectuó en el laboratorio con un estereoscopio. Para la identificación se utilizaron claves taxonómicas (Roldán 1988, Merritt y Cummins 1996, Carrera y Fierro 2001, Springer et al. 2010 y Springer et al. en prep.). El material fue depositado en el Museo de Insectos, Centro Zamorano de Biodiversidad, Escuela Agrícola Panamericana, Honduras.

Cuadro 1. Caracterización de las tres estaciones de muestreo de macroinvertebrados acuáticos en la quebrada Santa Inés, Valle del Yeguare, Honduras.

\begin{tabular}{|c|c|c|c|}
\hline $\begin{array}{l}\text { Esta- } \\
\text { ción }\end{array}$ & $\begin{array}{l}\text { Coordena- } \\
\text { das }\end{array}$ & $\begin{array}{l}\text { Altitud } \\
\text { (msnm) }\end{array}$ & Vegetación \\
\hline Alta & $\begin{array}{l}86^{\circ} 56^{\prime} 27.43^{\prime \prime} \\
13^{\circ} 57^{\prime} 39.04^{\prime \prime}\end{array}$ & 1,071 & $\begin{array}{lr}\text { Bosque de } & \text { pino } \\
\text { (Pinus spp.), pero hay } \\
\text { árboles } & \text { frutales } \\
\text { introducidos, } & \\
\text { especialmente } & \text { de } \\
\text { manzana } & \text { rosa } \\
\text { (Syzygium jambos) } & \text { cerca de la quebrada }\end{array}$ \\
\hline Media & $\begin{array}{l}86^{\circ} 56^{\prime} 58.52^{\prime \prime} \\
13^{\circ} 57^{\prime} 57.19^{\prime \prime}\end{array}$ & 1,002 & $\begin{array}{lr}\text { Introducida, } & \text { hay } \\
\text { guamas (Inga } & \text { spp.), } \\
\text { manzana } & \text { rosa, } \\
\text { guácimo rojo } & \text { (Luehea } \\
\text { seemani), } & \text { algunos } \\
\text { Pinus y } & \text { varias } \\
\text { asteráceas } & \end{array}$ \\
\hline Baja & $\begin{array}{l}86^{\circ} 57^{\prime} 46.07^{\prime \prime} \\
13^{\circ} 58^{\prime} 40.23^{\prime \prime}\end{array}$ & 846 & $\begin{array}{l}\text { Bosque de pino } \\
\text { (Pinus spp.) }\end{array}$ \\
\hline
\end{tabular}

Se graficó el número acumulado de géneros $S(n)$, recolectados en las estaciones, en relación al esfuerzo de muestreo (n). Posteriormente se calculó la riqueza de especies a través del índice de Chao (Teillier et al. 2004). Para determinar la estructura numérica de la comunidad de macroinvertebrados en los tres sitios muestreasdos se utilizó el índice de Shannon-Weaver (H') y el índice alfa de Fisher (S). Para estimar la abundancia de las especies comunes en las estaciones se usó la dominancia de Simpson (D) y Berger-Parker (BP). La riqueza de la comunidad se estimó mediante la Riqueza de Margalef (R) y de Menhinick (M). La equidad entre comunidades se determinó mediante Pielou (E). Por último, la similitud entre las comunidades de las estaciones muestreadas se estimó mediante el índice de Similitud de Sørensen (C) (Brower et al. 1997). Para determinar la estructura trófica de los macroinvertebrados acuáticos en los tres sitios muestreados de la quebrada se asignó cada una de las familias a un grupo funcional. Para lo anterior se utilizó la clasificación basada en Lampert y Sommer (2007), en donde los macroinvertebrados se dividen en cuatro grupos de acuerdo a sus relaciones alimentarias: fragmentadores, filtradores, raspadores y depredadores. Los fragmentadores se alimentan de materia orgánica particulada. Los filtradores se alimentan de materia orgánica particulada fina o muy fina, mientras que los raspadores se alimentan de algas del perifiton (Lampert y Sommer 2007).

\section{Resultados}

Se recolectaron 3,525 individuos en los tres sitios y se clasificaron en 104 taxa. De los individuos recolectados, se identificaron cuatro filos, ocho clases y 17 órdenes. Los poliquetos (Polychaeta) solo se identificaron a nivel de la clase, mientras que los hirudíneos a nivel de orden (Glossiphoniiformes). El resto de los individuos se identificaron a nivel de familia (55) (Cuadro 2). Se determinaron 79 géneros de macroinvertebrados acuáticos, aunque no fue posible identificar a este nivel a los miembros de 19 familias (No determinado, Cuadro 2). Ephemeroptera fue el orden más abundante con $33.8 \%$ de los individuos registrados, seguido por Hemiptera con $27.3 \%$ y Odonata con $18.0 \%$ (Figura 2). La familia con mayor número de individuos fue Naucoridae (Hemiptera) con $14.3 \%$ del total (506 individuos). Por otro lado, Coleoptera, Hemiptera y Trichoptera estuvieron representados por ocho familias cada uno, mientras que Megaloptera, Lepidoptera y Blatodea, entre otros, estuvieron representados por una única familia. El género dominante en la microcuenca fue Limnocoris (Naucoridae) con 346 individuos. 
Cuadro 2. Macroinvertebrados acuáticos, individuos por taxa, recolectados en la quebrada Santa Inés, Departamentos de Francisco Morazán y El Paraíso, Honduras.

\begin{tabular}{|c|c|c|c|c|c|}
\hline Phylum & Clase & Orden & Familia & Género, tribu o subfamilia & Individuos \\
\hline Annelida & Hirudinea & Glossiphoniiformes & No determinada & No determinado & 1 \\
\hline Annelida & Polychaeta & No determinado & No determinada & No determinado & 1 \\
\hline Arthropoda & Arachnoidea & Acari & Limnesiidae & No determinado & 1 \\
\hline Arthropoda & Crustacea & Brachyura & Pseudothelphusidae & No determinado & 3 \\
\hline Arthropoda & Insecta & Blattodea & Blaberidae & Epilampra & 2 \\
\hline Arthropoda & Insecta & Coleoptera & Dryopidae & Dryops & 1 \\
\hline Arthropoda & Insecta & Coleoptera & Dryopidae & Helichus & 3 \\
\hline Arthropoda & Insecta & Coleoptera & Dryopidae & Pelonomus & 11 \\
\hline Arthropoda & Insecta & Coleoptera & Dysticidae & No determinado & 10 \\
\hline Arthropoda & Insecta & Coleoptera & Elmidae & No determinado & 2 \\
\hline Arthropoda & Insecta & Coleoptera & Elmidae & Heterelmis & 7 \\
\hline Arthropoda & Insecta & Coleoptera & Elmidae & Hexanchorus & 2 \\
\hline Arthropoda & Insecta & Coleoptera & Elmidae & Macrelmis & 5 \\
\hline Arthropoda & Insecta & Coleoptera & Elmidae & Microcylloepus & 6 \\
\hline Arthropoda & Insecta & Coleoptera & Elmidae & Neocylloepus & 6 \\
\hline Arthropoda & Insecta & Coleoptera & Elmidae & Phanocerus & 4 \\
\hline Arthropoda & Insecta & Coleoptera & Elmidae & Xenelmis & 1 \\
\hline Arthropoda & Insecta & Coleoptera & Gyrinidae & Gyretes & 39 \\
\hline Arthropoda & Insecta & Coleoptera & Hydrophilidae & No determinado & 5 \\
\hline Arthropoda & Insecta & Coleoptera & Psephenidae & Psephenops & 11 \\
\hline Arthropoda & Insecta & Coleoptera & Ptilodactylidae & Anchytarsus & 11 \\
\hline Arthropoda & Insecta & Coleoptera & Staphylinidae & No determinado & 1 \\
\hline Arthropoda & Insecta & Diptera & Chironomidae & Chironominii & 7 \\
\hline Arthropoda & Insecta & Diptera & Chironomidae & Orthocladiinae & 22 \\
\hline Arthropoda & Insecta & Diptera & Chironomidae & Tanypodinae & 16 \\
\hline Arthropoda & Insecta & Diptera & Chironomidae & Tanytarsini & 3 \\
\hline Arthropoda & Insecta & Diptera & Culicidae & Culex & 1 \\
\hline Arthropoda & Insecta & Diptera & Dixidae & Dixella & 5 \\
\hline Arthropoda & Insecta & Diptera & Simulidae & Simulium & 32 \\
\hline Arthropoda & Insecta & Diptera & Tabanidae & Chrysops & 5 \\
\hline Arthropoda & Insecta & Diptera & Tipulidae & No determinado & 1 \\
\hline Arthropoda & Insecta & Diptera & Tipulidae & Hexatoma & 2 \\
\hline Arthropoda & Insecta & Ephemeroptera & Baetidae & Americabaetis & 22 \\
\hline Arthropoda & Insecta & Ephemeroptera & Baetidae & Baetodes & 4 \\
\hline
\end{tabular}


López et al.: Macrofauna Acuática de la Quebrada Santa Inés, Subcuenca del Río Yeguare, Honduras

Cuadro 2. Continuación.

\begin{tabular}{|c|c|c|c|c|c|}
\hline Phylum & Clase & Orden & Familia & Género, tribu o subfamilia & Individuos \\
\hline Arthropoda & Insecta & Ephemeroptera & Baetidae & Callibaetis & 167 \\
\hline Arthropoda & Insecta & Ephemeroptera & Baetidae & Camelobaetidius & 51 \\
\hline Arthropoda & Insecta & Ephemeroptera & Baetidae & Cloeodes & 33 \\
\hline Arthropoda & Insecta & Ephemeroptera & Baetidae & No determinado & 78 \\
\hline Arthropoda & Insecta & Ephemeroptera & Baetidae & Mayobaetis & 6 \\
\hline Arthropoda & Insecta & Ephemeroptera & Baetidae & Moribaetis & 23 \\
\hline Arthropoda & Insecta & Ephemeroptera & Caenidae & Caenis & 15 \\
\hline Arthropoda & Insecta & Ephemeroptera & Heptageniidae & Epeorus & 48 \\
\hline Arthropoda & Insecta & Ephemeroptera & Isonychiidae & Isonychia & 52 \\
\hline Arthropoda & Insecta & Ephemeroptera & Leptohyphidae & Allenhyphes & 1 \\
\hline Arthropoda & Insecta & Ephemeroptera & Leptohyphidae & Leptohyphes & 44 \\
\hline Arthropoda & Insecta & Ephemeroptera & Leptohyphidae & Tricorythodes & 213 \\
\hline Arthropoda & Insecta & Ephemeroptera & Leptohyphidae & Vacuperinus & 1 \\
\hline Arthropoda & Insecta & Ephemeroptera & Leptophlebiidae & Choroterpes & 1 \\
\hline Arthropoda & Insecta & Ephemeroptera & Leptophlebiidae & Farrodes & 179 \\
\hline Arthropoda & Insecta & Ephemeroptera & Leptophlebiidae & Thraulodes & 252 \\
\hline Arthropoda & Insecta & Hemiptera & Belostomatidae & Belostoma & 172 \\
\hline Arthropoda & Insecta & Hemiptera & Gerridae & No determinado & 11 \\
\hline Arthropoda & Insecta & Hemiptera & Hebridae & Hebrus & 3 \\
\hline Arthropoda & Insecta & Hemiptera & Hydrometridae & Hydrometra & 1 \\
\hline Arthropoda & Insecta & Hemiptera & Naucoridae & Ambrysus & 92 \\
\hline Arthropoda & Insecta & Hemiptera & Naucoridae & No determinado & 65 \\
\hline Arthropoda & Insecta & Hemiptera & Naucoridae & Limnocoris & 348 \\
\hline Arthropoda & Insecta & Hemiptera & Naucoridae & Pelocoris & 1 \\
\hline Arthropoda & Insecta & Hemiptera & Notonectidae & No determinado & 7 \\
\hline Arthropoda & Insecta & Hemiptera & Notonectidae & Notonecta & 46 \\
\hline Arthropoda & Insecta & Hemiptera & Ochteridae & Ochterus & 1 \\
\hline Arthropoda & Insecta & Hemiptera & Veliidae & Rhagovelia & 214 \\
\hline Arthropoda & Insecta & Lepidoptera & Crambidae & Petrophila & 9 \\
\hline Arthropoda & Insecta & Megaloptera & Corydalidae & Corydalus & 11 \\
\hline Arthropoda & Insecta & Megaloptera & Corydalidae & Platyneuromus & 1 \\
\hline Arthropoda & Insecta & Odonata & Calopterygidae & Hetaerina & 98 \\
\hline Arthropoda & Insecta & Odonata & Coenagrionidae & Acanthagrion & 4 \\
\hline Arthropoda & Insecta & Odonata & Coenagrionidae & Argia & 277 \\
\hline Arthropoda & Insecta & Odonata & Coenagrionidae & No determinado & 1 \\
\hline
\end{tabular}


Cuadro 2. Continuación.

\begin{tabular}{|c|c|c|c|c|c|}
\hline Phylum & Clase & Orden & Familia & Género, tribu o subfamilia & Individuos \\
\hline Arthropoda & Insecta & Odonata & Coenagrionidae & Leptobasis & 1 \\
\hline Arthropoda & Insecta & Odonata & Coenagrionidae & Nehalennia & 3 \\
\hline Arthropoda & Insecta & Odonata & Gomphidae & Epigomphus & 31 \\
\hline Arthropoda & Insecta & Odonata & Gomphidae & Erpetogomphus & 5 \\
\hline Arthropoda & Insecta & Odonata & Gomphidae & Phyllogomphoides & 4 \\
\hline Arthropoda & Insecta & Odonata & Gomphidae & Progomphus & 26 \\
\hline Arthropoda & Insecta & Odonata & Lestidae & Archilestes & 49 \\
\hline Arthropoda & Insecta & Odonata & Libellulidae & Brechmorhoga & 56 \\
\hline Arthropoda & Insecta & Odonata & Libellulidae & Dythemis & 18 \\
\hline Arthropoda & Insecta & Odonata & Libellulidae & $E \lg a$ & 3 \\
\hline Arthropoda & Insecta & Odonata & Libellulidae & No determinado & 8 \\
\hline Arthropoda & Insecta & Odonata & Libellulidae & Macrothemis & 28 \\
\hline Arthropoda & Insecta & Odonata & Libellulidae & Micrathyria & 4 \\
\hline Arthropoda & Insecta & Odonata & Libellulidae & Orthemis & 1 \\
\hline Arthropoda & Insecta & Odonata & Libellulidae & Pseudoleon & 1 \\
\hline Arthropoda & Insecta & Odonata & Platystitidae & Palaemnema & 16 \\
\hline Arthropoda & Insecta & Plecoptera & Perlidae & Anacroneuria & 82 \\
\hline Arthropoda & Insecta & Trichoptera & Glossosomatidae & No determinado & 2 \\
\hline Arthropoda & Insecta & Trichoptera & Helicopsychidae & Helicopsyche & 2 \\
\hline Arthropoda & Insecta & Trichoptera & Hydrobiosidae & Atopsyche & 4 \\
\hline Arthropoda & Insecta & Trichoptera & Hydropsychidae & Leptonema & 34 \\
\hline Arthropoda & Insecta & Trichoptera & Hydropsychidae & Macronema & 2 \\
\hline Arthropoda & Insecta & Trichoptera & Hydropsychidae & Smicridea & 6 \\
\hline Arthropoda & Insecta & Trichoptera & Hydroptilidae & Hydroptila & 15 \\
\hline Arthropoda & Insecta & Trichoptera & Hydroptilidae & Ochrotrichia & 4 \\
\hline Arthropoda & Insecta & Trichoptera & Hydroptilidae & Oxyethira & 2 \\
\hline Arthropoda & Insecta & Trichoptera & Leptoceridae & Oecetis & 1 \\
\hline Arthropoda & Insecta & Trichoptera & Philopotamidae & Chimarra & 68 \\
\hline Arthropoda & Insecta & Trichoptera & Polycentropodidae & Polycentropus & 19 \\
\hline Arthropoda & Insecta & Trichoptera & Polycentropodidae & Polyplectropus & 17 \\
\hline Mollusca & Gastropoda & Basommatophora & Ancylidae & No determinado & 7 \\
\hline Mollusca & Gastropoda & Basommatophora & Planorbiidae & No determinado & 8 \\
\hline Mollusca & Gastropoda & Mesogastropoda & Hydrobiidae & No determinado & 2 \\
\hline Mollusca & Bivalvia & Veneroida & Sphaeriidae & No determinado & 1 \\
\hline Platyhelmintes & Turbellaria & Tricladida & Planariidae & No determinado & 212 \\
\hline
\end{tabular}




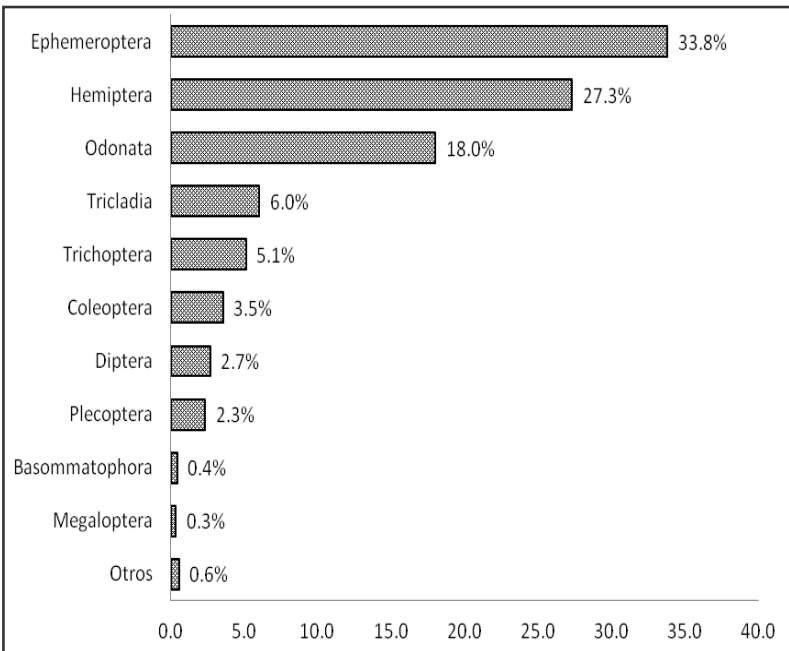

Figura 2. Porcentaje de individuos por orden de macroinvertebrados acuáticos encontrados en la quebrada Santa Inés, Honduras.

La aparición de géneros nuevos a lo largo de los muestreos se mantuvo oscilante en el tiempo (Figura 3). El mayor número de géneros fue 39 en el primer muestreo, mientras que en el último muestreo aparecieron cinco géneros que no habían sido encontrados (Figura 3). El valor del índice $\mathrm{H}^{\prime}$ para Santa Inés fue 3.55. Además, se estimó $S=20.11$ y Chao = 132 para los tres sitios muestreados como valores determinantes para la comunidad de macroinvertebrados en Santa Inés.

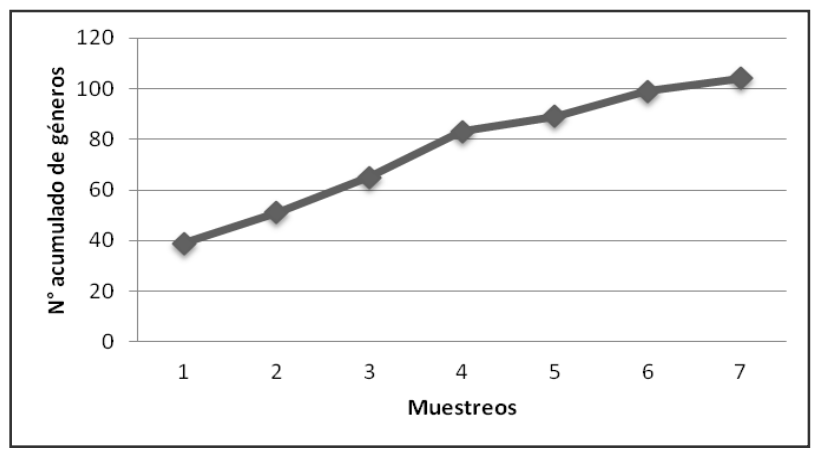

Figura 3. Acumulación de géneros de macroinvertebrados acuáticos en relación a los muestreos efectuados en tres sitios de la quebrada Santa Inés, Honduras.
El mayor número de individuos recolectados por estaciones fue en la alta $(1,535)$. De igual manera, en la alta se recolectó el mayor número de taxa $(82$, Cuadro 3). No existen diferencias entre los índices de diversidad calculados para las tres estaciones $\left(\mathrm{t}_{1}=\right.$ $\left.0,40, \mathrm{t}_{2}=1,16, \mathrm{t}_{3}=-1,43, \mathrm{p}>0,05\right)$. La correlación entre $\mathrm{H}^{\prime}$ y $\mathrm{S}$ fue de $0.05, \mathrm{P}>0.05$. La dominancia fue baja en las tres estaciones (Cuadro 3), mientras que, los máximos valores de $\mathrm{R}$ y $\mathrm{M}$ fueron para la alta $\mathrm{y}$ media, respectivamente (Cuadro 3). La E más alta fue para la estación media (Cuadro 3). Las estaciones alta y baja son las más similares con $\mathrm{C}=0.73$.

Cuadro 3. Valores de los índices de diversidad, dominancia, riqueza y equidad calculados para las tres estaciones muestreadas en tres sitios en la quebrada Santa Inés, Honduras.

\begin{tabular}{lrrr}
\hline \multirow{2}{*}{ Índice } & \multicolumn{3}{c}{ Estaciones } \\
\cline { 2 - 4 } & Alta & Media & Baja \\
\hline Shannon & 3.32 & 3.31 & 3.38 \\
Fisher & 18.51 & 15.79 & 17.33 \\
Simpson & 0.94 & 0.95 & 0.94 \\
Dominancia & 0.06 & 0.05 & 0.06 \\
Berger-Parker & 0.13 & 0.10 & 0.15 \\
Margalef & 11.04 & 9.21 & 10.21 \\
Menhinick & 2.10 & 2.18 & 2.15 \\
Pielou & 0.75 & 0.80 & 0.79 \\
$\mathrm{~N}^{\circ}$ géneros & 82 & 63 & 73 \\
$\mathrm{~N}^{\circ}$ Individuos & 1,535 & 838 & 1,152 \\
\hline
\end{tabular}

El grupo funcional dominante en los tres sitios fue el de los depredadores (55\%). Los filtradores fueron el segundo grupo dominante $(28 \%)$, mientras que el grupo de los raspadores constituyó 3\% (Figura 4). Las familias con dos tipos de alimentación dentro de los cuatro grupos funcionales representaron $6 \%$ (Figura 4). Por estaciones, se encontró que el grupo de los depredadores se mantiene casi constante a lo largo de la quebrada (Figura 5). El grupo de los fragmentadores fue mejor representado en la estación alta, al igual que los filtradores, mientras que los raspadores fueron más abundantes en la estación baja (Figura 5). 


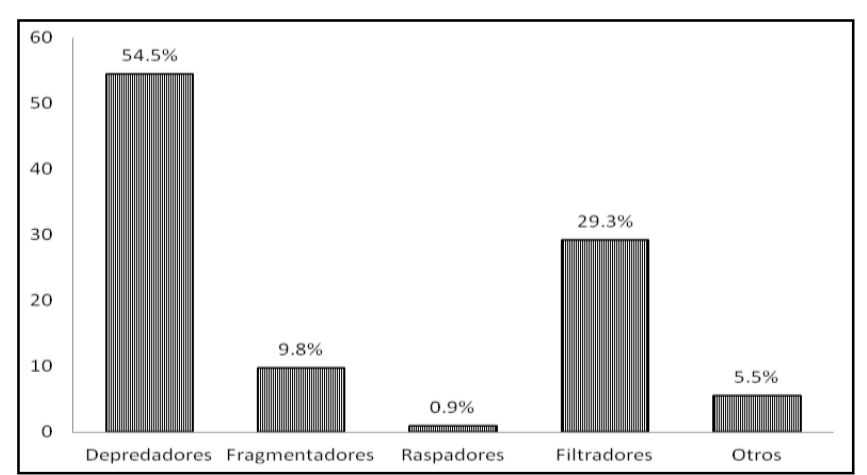

Figura 4. Porcentaje por grupo trófico funcional de los macroinvertebrados acuáticos encontrados en la quebrada Santa Inés, Honduras.

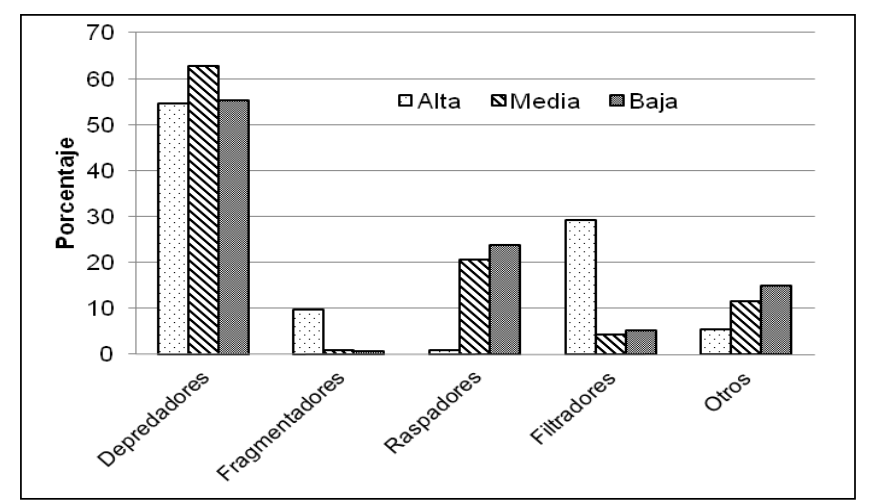

Figura 5. Porcentajes de individuos según su gremio trófico encontrados en cada una de las estaciones alta media y baja muestreadas en la quebrada Santa Inés, Honduras.

Algunos taxa fueron encontrados solamente en una de las tres estaciones muestreadas. En la estación alta se encontraron 17 taxa que no estuvieron presentes en las otras dos estaciones, tal es el caso de Allenhyphes (Ephemeroptera: Leptohyphidae) (Cuadro 4). En la media también se encontraron algunos macroinvertebrados que no se recolectaron en la alta ni en la baja, por ejemplo Helichus (Coleoptera: Dryopidae) y un género no determinado de la familia Naucoridae que fue muy abundante en esta estación (Cuadro 4). En la estación baja se encontraron nuevos taxa que no estuvieron en las otras dos estaciones tales como Neocylloepus (Coleoptera: Elmidae) y
Acanthagrion (Odonata: Coenagrionidae). Otros 19 taxa, como por ejemplo Epeorus (Ephemeroptera: Heptageniidae), se encontraron en la estación media y la baja, pero no en la alta.

Cuadro 4. Número de individuos (por género) de macroinvertebrados acuáticos presentes solamente en una de las tres estaciones muestreadas (alta, media o baja) en la quebrada Santa Inés, Honduras.

\begin{tabular}{|c|c|c|c|c|}
\hline Familia & Género & Alta & Media & Baja \\
\hline Coenagrionidae & Acanthagrion & & & 4 \\
\hline Coenagrionidae & No determinado & & & 1 \\
\hline Coenagrionidae & Leptobasis & 1 & & \\
\hline Corydalidae & Platyneuromus & 1 & & \\
\hline Culicidae & Culex & 1 & & \\
\hline Dryopidae & Dryops & & & 1 \\
\hline Dryopidae & Helichus & & 3 & \\
\hline Elmidae & Hexanchorus & 2 & & \\
\hline Elmidae & Neocylloepus & & & 6 \\
\hline Elmidae & Xenelmis & & 1 & \\
\hline Glossiphoniidae & No determinado & & & 1 \\
\hline Glossosomatidae & No determinado & & 2 & \\
\hline Hydrobiidae & No determinado & 2 & & \\
\hline Hydrometridae & Hydrometra & 1 & & \\
\hline Leptoceridae & Oecetis & & & 1 \\
\hline Leptohyphidae & Allenhyphes & 1 & & \\
\hline Leptohyphidae & Vacuperinus & & & 1 \\
\hline Leptophlebiidae & Chorotherpes & & 1 & \\
\hline Libellulidae & Micrathyria & 4 & & \\
\hline Libellulidae & Orthemis & 1 & & \\
\hline Libellulidae & Pseudoleon & 1 & & \\
\hline Lymnessiidae & No determinado & 1 & & \\
\hline Naucoridae & No determinado & & 65 & \\
\hline Naucoridae & Pelicoris & & & 1 \\
\hline Notonectidae & No determinado & 7 & & \\
\hline Ochteridae & Ochterus & & 1 & \\
\hline Planorbiidae & No determinado & 8 & & \\
\hline Polychaeta & No determinado & 1 & & \\
\hline Pseudothelpusidae & No determinado & & & 3 \\
\hline Sphaeriidae & No determinado & 1 & & \\
\hline Staphylinidae & No determinado & 1 & & \\
\hline Tipulidae & No determinado & 1 & & \\
\hline
\end{tabular}




\section{Discusión}

La calidad del agua, la disponibilidad de alimento y las características del sustrato son los factores que tienden a determinar la abundancia y distribución de los macroinvertebrados acuáticos (Merritt y Cummins 1996, Orive y Rallo 2002). Las estaciones evaluadas son ricas en detritus proveniente de materiales como hojas y troncos que caen al agua. Este detritus, que constituye una fuente importante de alimento, determina la abundancia de algunos grupos de macroinvertebrados acuáticos. La variedad de microhábitats en la quebrada, también contribuye a la abundancia y distribución de los macroinvertebrados acuáticos. Además, el contenido de materia orgánica en el sedimento y el oxígeno disuelto contribuyen a determinar la estructura de la comunidad en un hábitat determinado (Lampert y Sommer 2007, Jacobsen et al. 2003). Todos estos factores son los responsables directos de la distribución y abundancia de los macroinvertebrados acuáticos en la Quebrada Santa Inés. La heterogeneidad en la comunidad de macroinvertebrados en los tres sitios muestreados está determinada por la heterogeneidad en el tipo de sustratos presentes en las estaciones muestreadas (Pérez y Segnini 2005).

Santa Inés es una quebrada de montaña a una altitud promedio de 1,385 msnm. En este tipo de aguas hay grupos de macroinvertebrados que tienden a ser dominantes, como los miembros de Ephemeroptera y Trichoptera (Roldán 1988). Estos órdenes predominan en corrientes de aguas transparentes, oligotróficas, frías y bien oxigenadas (Toro et al. 2003). Lo anterior explica la abundancia de efemerópteros en los sitios muestreados (Figura 2). Grupos como Megaloptera, Lepidoptera y Blattodea se encontraron en menor cantidad (Figura 2), lo cual según Roldán (1999) es lo que tiende a suceder en los ríos de montaña. La abundancia de individuos del género Limnocoris (Hemiptera: Naucoridae), está relacionada con los tipos de sustratos presentes en las estaciones muestreadas. Limnocoris vive en remansos de ríos y quebradas, adheridos a troncos, ramas y piedras e incluso hay especies que se entierran en el suelo arenoso (Roldán 1988, 2003), de aquí su abundancia en los sitios muestreados en la quebrada Santa Inés.
La curva de acumulación de géneros de macroinvertebrados de la quebrada Santa Inés no se estabilizó (Figura 3). Por lo tanto, aunque el número de géneros por muestreo tiende a disminuir, es necesario un mayor esfuerzo de muestreo para la identificación de géneros adicionales.

En los dos índices de diversidad calculados para las tres estaciones, los resultados son similares, el valor más bajo se determinó para la estación media (Cuadro 3). Hay que recordar que las comunidades de macroinvertebrados acuáticos tienden a cambiar su riqueza y abundancia de acuerdo a la perturbación (Bryce y Hughes 2003). Los resultados sugieren que los factores antropogénicos que afectan el agua en la quebrada Santa Inés son mínimos. Así mismo, se determinó un valor relativamente alto de los índices de diversidad, lo cual es indicativo de aguas claras y limpias (Torres et al. 2006). Al contrario, valores bajos de diversidad están relacionados con la contaminación del agua. La diversidad y abundancia de grupos como Ephemeroptera y Trichoptera en las estaciones muestreadas ratifican estas observaciones (López 2008).

En la composición trófica en los tres sitios muestreados, el grupo de los depredadores fue en general el más abundante, al igual fue el grupo más representativo en las tres estaciones. Esto sugiere que en la quebrada existe alimento disponible en abundancia. La teoría del continuo del río (Vannote et al. 1980) explica cómo los organismos se distribuyen en las corrientes de acuerdo a las características del agua, la disponibilidad de luz, la oferta de alimento y la presencia de depredadores, entre otros factores (Bernal et al. 2006, Lampert y Sommer 2007). El concepto del continuo rivereño, además, explica que el grupo de los depredadores tiende a ser constante a lo largo del río, lo cual se ajusta a lo observado en Santa Inés (Figura 5). Al contrario, grupos tales como los fragmentadores son más abundantes en las partes altas de los ríos o bien en ríos de primer orden, debido a que es ahí donde hay más aporte de hojarasca o materia particulada gruesa. Grupos tales como los filtradores que también tienden a ser abundantes en las partes altas de los ríos, aumentan proporcionalmente en las partes bajas o desembocaduras, debido a la abundancia de alimento o materia particulada fina, 
como consecuencia de la dinámica física de los sistemas lóticos (Pérez et al. 2004).

Los fragmentadores disminuyeron aguas abajo, mientras que los filtradores, abundantes en la estación alta, disminuyeron en la estación media y baja (Figura 5). Las características físicoquímicas del agua podrían estar influyendo en el reemplazo de los filtradores por familias de otros hábitos alimentarios en la estación media y baja. Los raspadores tienden a ser común en las partes medias de los ríos, donde hay más algas (Lampert y Sommer 2007). Por lo general, los organismos de hábitos raspadores tienden a reemplazar a los fragmentadores, particularmente por aquellos que pueden fijarse a los substratos (Pérez et al. 2004). En la media y baja, los filtradores disminuyeron con respecto a lo esperado, aunque fueron más abundantes que los fragmentadores en ambas estaciones. No obstante, de igual manera, las características físicas o químicas de la corriente podrían afectar la presencia de este grupo trófico en la estación media y baja. Además, los filtradores tienden a disminuir con algún tipo de perturbación en el agua (Lampert y Sommer 2007). Las estaciones media y baja están sometidas a actividades antrópicas cercanas, por ejemplo, en la baja hay una toma de agua para riego y otras actividades y al lado de la estación media hay dos viviendas que descargan aguas servidas en la quebrada. Por lo tanto, varios grupos desaparecen debido a factores ambientales o antrópicos que afectan la corriente.

Debido a lo anterior, se determina la presencia de ciertas familias de organismos en unos sitios y su reemplazo por familias diferentes en otros sitios, pero que cumplen la misma función en el sistema (Bernal et al. 2006). Aparentemente esta sustitución es la que se está dando en Santa Inés. Por ejemplo, 19 taxa que se encontraron en la estación alta, desaparecen en las otras dos estaciones, como Allenhyphes (Ephemeroptera: Leptohyphidae), Hydrometra (Hemiptera: Hydrometridae) y Platyneuromus (Megaloptera: Corydalidae). Al contrario, géneros como Macrelmis (Coleoptera: Elmidae) Chrysops (Diptera: Tabanidae) y Epeorus (Ephemeroptera: Heptageniidae) se encontraron en la estación media y baja, pero no en la alta.

El agua en la quebrada Santa Inés está afectada por varios agentes que probablemente han alterado la composición de la comunidad de macroinvertebrados acuáticos en el sitio. No obstante, la biodiversidad de la quebrada Santa Inés es alta en comparación con otros sistemas similares estudiados (Roldán 1992). Este sistema contiene una alta complejidad estructural, donde se encuentran representados todos los grupos tróficos de macroinvertebrados acuáticos posibles en un sistema ribereño. La geografía de la microcuenca, el uso actual del suelo y la comunidad de macroinvertebrados acuáticos que se encuentra en la quebrada la hacen un sujeto ideal de estudio bajo el concepto del continuo ribereño.

\section{Literatura Citada}

Alba-Tercedor, J. 1996. Macroinvertebrados acuáticos y calidad de las aguas de los ríos. Memorias IV Simposio del Agua en Andalucía. Almeria 2: 203-213.

Arcos, I. 2005. Efecto del ancho los ecosistemas riparios en la conservación de la calidad del agua y la biodiversidad en la microcuenca del Río Sesesmiles, Copán, Honduras. Tesis de Maestría en Manejo Integrado de Cuencas Hidrográficas. CATIE. 104 p.

Bass, D. 1993. A preliminary survey of stream invertebrates from Guanaja Island, Bay Islands, Honduras. Brenesia 40:175 179.

Bernal, E., D. García, M.A. Novoa y A. Pinzón. 2006. Caracterización de la comunidad de macroinvertebrados de la quebrada Paloblanco de la cuenca del Río Otún (Risaralda, Colombia). Acta Biol Colomb. 11:45-59.

Blahnik, J.R. y R.W. Holzenthal. 2008. Revision of the Mexican and Central American species of Mortoniella (Trichoptera: Glossosomatidae: Protoptilinae). Zootaxa 1711:1-72.

Brower, J.E., J.H. Zar y C.N. von Ende. 1997. Field and Laboratory Methods for General Ecology. WCB/McGraw-Hill. Boston, Estados Unidos. 273 p.

Bryce, S.A. y R.M. Hughes. 2003. Variable assemblage responses to multiple disturbance gradients: Case studies in Oregon and Appalachia, USA, p. 539-560. In: T.P. Simon (eds). Biological response signatures indicator patterns using aquatic communities. CRC Press, Boca Raton, Estados Unidos.

Carrera, C. y K. Fierro. 2001. Los macroinvertebrados acuáticos como indicadores de la calidad del agua: Manual de monitoreo. EcoCiencia. Quito, Ecuador. 67 p. 
López et al:: Macrofauna Acuática de la Quebrada Santa Inés, Subcuenca del Río Yeguare, Honduras

Cruz, M.E., G.A. Borjas, H.O. Martínez y O.O. Martínez. 1987. Parámetros Físico-Químicos Asociados a Macroinvertebrados Bentónicos en el Río Choluteca y sus Cabeceras. Tesis de Licenciatura. Universidad Nacional de Honduras. 117 p.

Estévez, A. y T.J. Polhemus. 2006. The small species of Belostoma (Heteroptera: Belostomatidae): Revision of plebejum group. Revista de Biología Tropical 55:147155.

Fenoglio, S., B.O. Tiziano, A. Czekaj y E. Rooeciszewska. 2008. Feeding habits, fine structure and microhabitat preference of Euthyplocia hecuba (Hagen, 1861) (Ephemeroptera: Euthyplociidae) nymphs from Honduras. Folia Biol. 56: 43-49.

García, L.A. y F. Jiménez. 2006. Efectos del bosque ribereño y de las actividades antrópicas en las características físico-químicas y en poblaciones de macroinvertebrados acuáticos en la subcuenca del río Tascalapa, Honduras. Recursos Naturales y Ambiente 48:35-46.

Harris, S. y R. Holzenthal. 1999. Hydroptilidae (Trichoptera) of Costa Rica: the genus Hydroptila Dalman. Stud Neotrop Fauna y Environm. 34:16-51.

Helawell, J.M. 1986. Biological indicators of freshwater pollution and environmental management. Elsevier Applied Sci. Publ. Londres. 546 p.

Herrera, B. 2001. Valoración económica del agua en la microcuenca Santa Inés, San Antonio de Oriente, Honduras. Tesis de Licenciatura. Zamorano. Honduras. $67 \mathrm{p}$.

Jacobsen J., S. Rostgaard y J.J. Vásconez. 2003. Are macroinvertebrate in high altitude streams affected by oxygen deficiency? Freshwater Biology 48: 20252032.

Lampert, W. y U. Sommer. 2007. Limnoecology: The ecology of lakes and streams. $2^{\mathrm{a}} \mathrm{Ed}$. Oxford University Street. Nueva York. 324 p.

López, L.I. 2008. Análisis y valoración de cuatro índices bióticos mediante la utilización de macro-invertebrados acuáticos en la subcuenca del Yeguare, Honduras. Tesis de Maestría en Biología. Universidad de Costa Rica. 150 p.

Merritt, R.W. y K.W. Cummins (eds.). 1996. An introduction to the aquatic insects of North America. Kendall/Hunt, Dubuque, Iowa, Estados Unidos. 862 p.

Molina, C.I., F.M. Gibon, J. Pinto y C. Rosales. 2008. Estructura de macro-invertebrados acuáticos en un río altoandino de La Cordillera Real, Bolivia: Variación anual y longitudinal en relación a factores ambientales. Ecología Aplicada 7:104-116.
Orive, E. y A. Rallo. 2002. Ríos de Bizkaia. Instituto de Estudios Territoriales de Bizkaia. Estudios Gráficos ZURE, S.A. 258 p.

Packer, J. 1966. A preliminary study of the mayflies of Honduras. Ceiba 12:1-10.

Pérez, B. y S. Segnini 2005. Variación espacial de la composición y diversidad de géneros del orden Ephemeroptera (Insecta) en un río tropical altoandino. Entomotrópica 20: 49-57.

Pérez, R.M., R.F. Pineda y V. Campos. 2004. Estructura trófica de las asociaciones de macroinvertebrados acuáticos de manantiales cársticos en la Huasteca Mexicana. Biológicas 6:37-47.

Prather, A. 2003. Revision of the neotropical caddisfly genus Phylloicus (Trichoptera: Calamoceratidae). Zootaxa 214:1- 214

Ramachandra, T.V. y M. Solanki. 2007. Ecological assessment of lentic water bodies of Bangalore. ENVIS Technical Report 25:1-105.

Renderos, M. E. 2001. Estudio exploratorio del potencial turístico de la cuenca Santa Inés, en el valle del Yeguare, Honduras. Tesis de Licenciatura. Zamorano. Honduras. 70 p.

Resh, V.H., M.J. Myers y M.J. Hannaford. 1996. Macroinvertebrates as Biotic Indicators of Environmental Quality, p. 647-667. In: F.R. Hauer y G.A. Lamberti (eds.). Stream Ecology. Academic Press. San Diego, California.

Roldán, G. 1988. Guía para el estudio de los macroinvertebrados acuáticos del Departamento de Antioquia. FEN Colombia. Bogotá, Colombia. 217 p.

Roldán, G. 1992. Fundamentos de limnología neotropical. Universidad de Antioquia. Medellín, Colombia. 529 p.

Roldán, G. 1999. Los macroinvertebrados y su valor como indicadores de la calidad del agua. Rev. Acad. Colomb. Cienc. 23:375-387.

Roldán, G. 2003. Bioindicación de la calidad del agua en Colombia: Uso del método BMWP/Col. Universidad de Antioquia. Colombia. 169 p.

Rosenberg, D.M., H.V. Danks y D.M. Lehmkuhl. 1986. Importance of insects in enviromental impact assesment. Envir. Manage. 10:773-783.

Smock, L.A. 1996. Macroinvertebrates Movements: Drift, Colonization, and Emergence, p. 371-390. In: F.R. Hauer y G.A. Lamberti (eds.). Stream Ecology. Academic Press. San Diego, California.

Springer, M. A. Ramírez y P. Hanson (eds.). 2011. Macroinvertebrados de agua dulce de Costa Rica; Volumen I: Rev. Biol. Trop. 
Springer, M. A. Ramírez y P. Hanson (eds.). Macroinvertebrados de agua dulce de Costa Rica; Volumen II: Revista de Biología Tropical (En preparación)

Teillier, S., A. Prina, G. Alfonso y F. Luebert. 2004. Aporte al conocimiento de la flora de los Andes del suroeste del Departamento de Malargüe, Argentina. Chloris Chilensis, Año 7. $\mathrm{N}^{\mathrm{o}}$ 1. Internet Webpage: http://www.chlorischile. cl. (Consultada: febrero 2011)

Toro, J., J.P. Schuster, J. Kurosawa. E. Araya y M. Contreras. 2003. Diagnóstico de la calidad del agua en sistemas lóticos utilizando diatomeas y macroinvertebrados bentónicos como bioindicadores Río Maipo (Santiago: Chile). Sociedad Chilena de ingeniería Hidráulica. Memorias XVI Congreso Chileno de Ingeniería Hidráulica: 1-11.

Torres, Y., G. Roldán, S. Asprilla y T.S. Rivas. 2006. Estudio preliminar de algunos aspectos ambientales y ecológicos de las comunidades de peces y macroinvertebrados acuáticos en el Río Tutunendo, Chocó, Colombia. Rev. Acad. Colomb. Cienc. 30:6776.
Vannote, R.L., G.W. Minshall, K.W. Cummins, J.R. Sedell y C.E. Cushing. 1980. The river continuum concept. Can. J. Fish. Aquat. Sci. 37: 130-137.

Vidal, A. 1930. Zancudos Anopheles de Honduras. Rev. Med. Hond. 1:439.

Walteros-Rodríguez, J.M. y J.E. Paiba-Alzate. 2010. Estudio preliminar de la comunidad de macroinvertebrados acuáticos en La Reserva Forestal Torre Cuatro. Bol.cient.mus.hist.nat. 14: 137-149.

Wiersema, N. A. y W. McCafferty. 2000. Generic revision of the North and Central American Leptohyphidae (Ephemeroptera: Pannota). Transactions of the American Entomological Society 126:337-371.

Recibido para publicación el 13 de enero de 2011.

Aceptado para publicación el 19 de noviembre de 2011. 